\title{
Ultimate regime of convection: Robustness to poor thermal reservoirs
}

\author{
P.-E. Roche, F. Gauthier, B. Chabaud, and B. Hébral \\ Centre de Recherches sur les Très Basses Températures, Laboratoire du CNRS, associé à l'Université \\ Joseph Fourier, 25 avenue des Martyrs, BP166, F-38042 Grenoble Cedex 9, France
}

(Received 31 January 2005; accepted 26 July 2005; published online 30 November 2005)

\begin{abstract}
A transition to Kraichnan ultimate regime of convection has been reported in very high Rayleigh numbers experiments, but not in all of them. These apparently contradictory results can be explained by a recent phenomenological model that accounts for the nonideality of the plate thermal properties [Chillà et al., Phys. Fluids 16, 2452 (2004)]. In this paper, we present a direct test of this model, using a low conductivity plate. We found an unaltered transition, not compatible with the model's predictions. (C) 2005 American Institute of Physics. [DOI: 10.1063/1.2136807]
\end{abstract}

\section{MOTIVATION}

In 1962, Kraichnan predicted ${ }^{1}$ that a fully turbulent regime of convection will settle in Rayleigh-Bénard cells for an intense thermal driving force (very high Rayleigh number $\mathrm{Ra}$ ). This regime is characterized by turbulent boundary layers and consequently an enhanced heat transport efficiency (Nusselt number $\mathrm{Nu}$ ) compared to the other convection regimes. Kraichnan predicted a $\mathrm{Nu} \sim \mathrm{Ra}^{1 / 2}$ dependence, with a logarithmic correction that reduces the effective exponent right above the transition to this so-called ultimate regime.

Only five experiments and one numerical simulation have explored Rayleigh numbers at least at $\mathrm{Ra}=10^{13}$ and higher. For all of them, the Prandtl number was in the intermediate region $(0.7<\operatorname{Pr}<20)$. The experiments have been conducted nearly in the same conditions: cylindrical cells of aspect ratio 0.5 or 1 , cryogenic helium as the working fluid, copper top and bottom plates, and a thin stainless-steel side wall. These very high Rayleigh number studies can be separated into two sets according to the measured $\mathrm{Nu}(\mathrm{Ra})$ dependence. For two experiments, ${ }^{2,3}$ this dependence is smooth and follows approximately a 0.3 exponent power law, up to $\mathrm{Ra} \sim 10^{17}$ for one of them, ${ }^{3}$ while for two other experiments ${ }^{4,5}$ and the simulation ${ }^{6}$ the exponent increases from 0.3 to approximately 0.4 in the $R a=10^{11}-10^{12}$ region. This second regime has been interpreted as the Kraichnan ultimate regime, and this interpretation was strengthened by the measurement of the full 0.5 exponent in a rough surface cell. $^{7}$

No obvious parameter-such as the Prandtl number or the cell aspect ratio - seems to be correlated with the occurrence of the transition among these few studies. Therefore, understanding what can favor or suppress the occurrence of the ultimate regime remains today a major challenge for the understanding of very high Rayleigh number convection. ${ }^{8,9}$

Recently, Chillà and colleagues proposed a phenomenological model ${ }^{10}$ that tries to explain these apparently contradictory results. This model assesses the nonideality of the plates' thermal properties: if the conductivity and/or the heat capacity of the plates are not high enough, a constant temperature cannot be sustained at the fluid-plate interface during the development of thermal plumes. As a consequence, the global heat transfer is shown to saturate below the Kraichnan prediction. The Chillà-Rastello condition $(\mathrm{Cr})$ to observe the transition is

$$
\mathrm{Cr}>0.8 \text {, }
$$

where $\mathrm{Cr}$ is a plate quality factor that depends on the ratios of plate to fluid conductivities $\lambda_{p} / \lambda_{f}$, heights $a / h$ ( $a$ is the plate thickness) and heat capacities, as well as the usual dimensionless parameters $\mathrm{Nu}, \mathrm{Pr}$, aspect ratio $\Gamma$, and Reynolds number Re. For the cryogenic experiments previously mentioned, a thin plate and quasistatic approximations can be applied to the general expression of Cr. One gets ${ }^{10}$

$$
\mathrm{Cr} \simeq \frac{\pi^{2}}{\Gamma^{2}} \frac{a}{h} \frac{\lambda_{p}}{\lambda_{f}} \frac{1}{\operatorname{Re} \operatorname{Pr}} .
$$

For reference, we should mention two other recent studies of finite plate conductivity effect on convection, ${ }^{11,12}$ although they are not directly related to the ultimate regime issue. Some previous works are reported in these two papers.

In this paper, we present two experiments that have been conducted to test the Chillà $e t$ al. model. We took out of the shelves two 20-cm-high Rayleigh-Bénard cells in which the transition have been observed in the past: one with smooth surfaces ${ }^{4}$ and the other one with rough (corrugated) surfaces. ${ }^{7}$ Both bottom plates were removed and replaced with two others, geometrically similar but made out of brass: one with a smooth surface and the other with a rough one. The corresponding $\mathrm{Cr}$ parameter for these new bottom plates is smaller than 0.1 for $\mathrm{Ra}>10^{11}$. According to Chillà et al., the global heat transfer $\mathrm{Nu}(\mathrm{Ra})$ in the ultimate regime should be significantly reduced compared to the original all-copper experiments.

\section{EXPERIMENTAL SETUP AND RESULTS}

In order to make the comparison with the all-copper experiments straightforward, the experimental setup and the operating procedure are very similar to those of these previous studies. ${ }^{7,13}$ Since this experimental information is detailed in the previous papers, we shall only report here the differences with these experiments. 

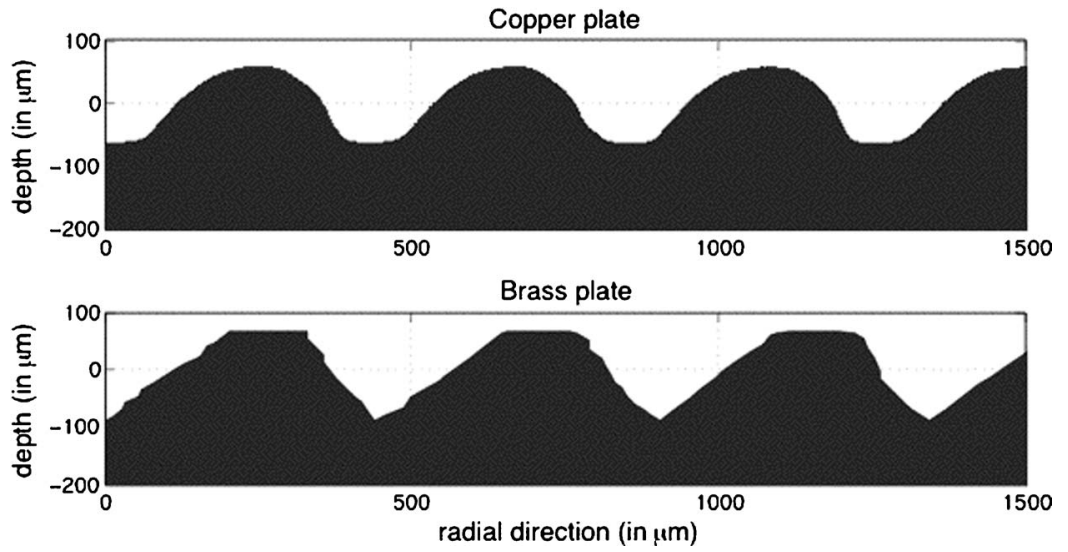

FIG. 1. Profilometer measurement of the roughness profile along a bottom plate diameter. Distances are given in micrometers. Upper plot: copper plate. Lower plot: brass plate.
The conduction of the brass plates has been measured with the inductive technique already used to measure the conductivity of the copper plates. ${ }^{14}$ We found $k_{\text {brass }}$ $=4.0 \mathrm{~W} \mathrm{~m}^{-1} \mathrm{~K}^{-1} \pm 10 \%$ at $4.2 \mathrm{~K}$, to be compared with $k_{\mathrm{Cu}}$ $=1090 \mathrm{~W} \mathrm{~m}^{-1} \mathrm{~K}^{-1}$ and $880 \mathrm{~W} \mathrm{~m}^{-1} \mathrm{~K}^{-1}$ at $4.2 \mathrm{~K}$ for the smooth surface and rough surface copper plates. In our experimental conditions, the heat capacity of brass is comparable to the one of copper. ${ }^{15}$ The surface roughness of the corrugated plate has been measured with a profilometer. The grooves are $450 \mu \mathrm{m}$ apart, ${ }^{16} 145 \mu \mathrm{m}$ deep, and look like the reference copper plate roughness (see Fig. 1). Both 25-mm-thick brass bottom plates are heated from below. In order to minimize the heater footprint on the other side of the plates, the heating wire has been uniformly (1.5 mm spacing) and carefully varnished on the bottom surface.

Special attention has been dedicated to the temperature measurements on the axis of the brass plates, near the fluidbrass interface. Finite element simulations ${ }^{17}$ have been used to optimize the shape of the copper thermometer holders. Such simulations allow to choose a quantitative compromise between the temperature field distortion and temperature drop correction (see below). Optimization was performed for $10^{9}<\mathrm{Ra}<10^{14}$ and by modeling the fluid's thermal boundary layer as a diffusive and spatially uniform slab of thickness $h / 2 \mathrm{Nu}$, where $h$ is the height of the cell. The thermometer holders are inserted into the brass plates, in long $\phi 4 \mathrm{~mm}$ holes ending with a $4 \mathrm{~mm}$ long $\phi 3 \mathrm{~mm}$ thread, itself ending $2 \mathrm{~mm}$ away from the surface in contact with the fluid (see Fig. 2). The holes and the thermometer holders are responsible for a local nonuniformity of temperature at the fluidbrass interface. According to the simulation, and for all the data presented in this paper, the magnitude of this effect is less than $1 \%$ of the temperature difference across the fluid. To this extent, the temperature measurement can be assumed as noninvasive. A correction needs to be applied to compensate for the temperature drop between the fluid-brass interface and the thermometer holders' effective positions. From the geometry of the thermometer holders' screw threads, this effective position can be estimated to be between 2 and $6 \mathrm{~mm}$ away from the fluid-brass interface. The simulations estimate the effective position to be $3.5 \mathrm{~mm}$ with a variation smaller than $4 \%$ as a function of $\mathrm{Nu}$. This $3.5 \mathrm{~mm}$ effective distance represents a $0.11 \mathrm{~K} / \mathrm{W}$ resistance correction. For all the data presented in this paper, this correction represents less than $20 \%$ of the temperature difference across the fluid (less than $10 \%$ for the smooth surface cell).

Temperature on both the top and bottom plates have been measured with doped Ge resistors, in situ calibrated (1) with respect to each other, (2) with respect to a commercially calibrated one, ${ }^{18}$ and (3) with respect to the fluid critical point. The estimated uncertainty on the temperature difference between the top and bottom plate is $1 \mathrm{mK}$. All the data presented have been obtained with a temperature difference across the fluid larger than $20 \mathrm{mK}$.

The arbitrarily Boussinesq criterion is that no fluid parameter should vary by more than $20 \%$ across the cell. Recent improvements in the knowledge of helium fluid properties ${ }^{19}$ have been taken into account in the calculation of $\mathrm{Ra}$ and $\mathrm{Nu}$. $\mathrm{Nu}$ has been corrected for the side wall conduction using the analytical correction formula presented in Ref. 20. The magnitude of this correction is nearly the same for all the cells. It decreases very rapidly with $\mathrm{Ra}$ and represents less than a $5 \%$ correction above $\mathrm{Ra}=10^{12}$.

Figures 3 and 4 show the heat transfer data in the rough and smooth surface "brass" experiments. The various solid

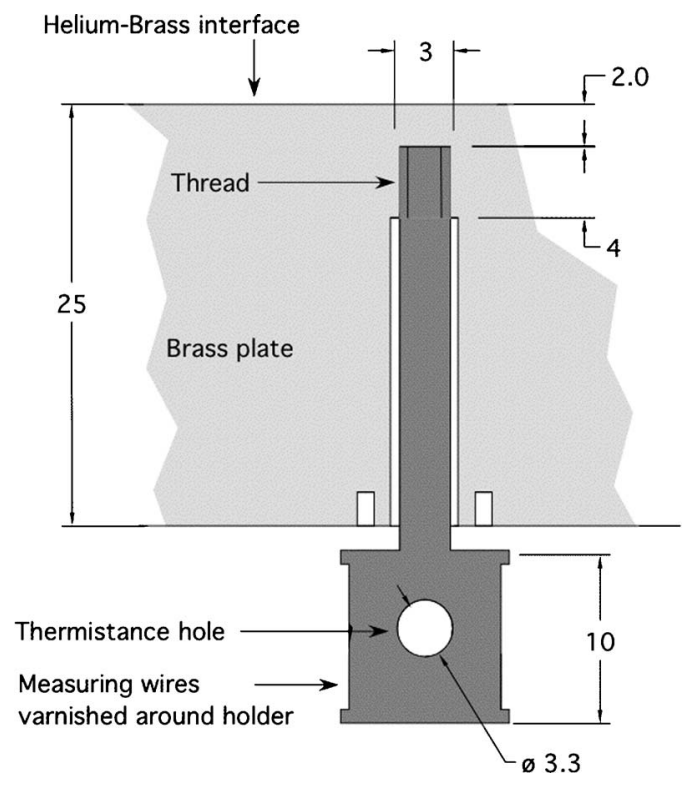

FIG. 2. Sketch of the copper thermometer holder (dark gray), screwed into the bottom brass plate (light gray). Distances are given in $\mathrm{mm}$. 


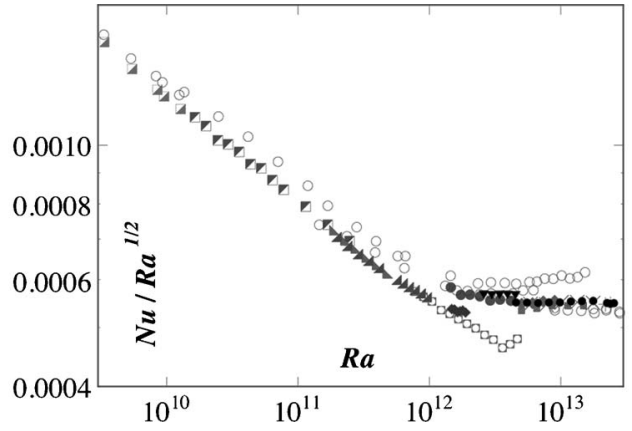

FIG. 3. Compensated Nusselt number $\mathrm{Nu} / \mathrm{Ra}^{1 / 2}$ (on a log scale) vs $\mathrm{Ra}$ for the rough surfaces experiments. Open circles: copper bottom plate. Other symbols: brass bottom plate.

symbols are associated with different data sets. Each set has been acquired for a fixed cell density and a fixed average temperature (within $3 \%$ for $\mathrm{Ra}>2 \times 10^{10}$ ) in the cell. The density and temperature of each set is chosen in the ranges $6.464-40.14 \mathrm{~kg} / \mathrm{m}^{3}$ and $4.673-6.000 \mathrm{~K}$. This experimental procedure was chosen to maintain the Prandtl number constant within each data set. The Prandtl numbers of the different sets are all in the $0.7<\operatorname{Pr}<5.4$ range. For comparison, the all-copper experiment data, restricted to the same Pr window, are also plotted (open circles).

In Fig. 3, the rough surface $\mathrm{Nu}$ data are compensated by $\mathrm{Ra}^{1 / 2}$ so that Kraichnan's $\mathrm{Nu}(\mathrm{Ra})$ power law corresponds to a plateau. Such plateaus are found for both the brass and the all-copper experiments, and-within experimental uncertainty - they fall on each other. Some extra data between $\mathrm{Ra}=3 \times 10^{13}$ and $\mathrm{Ra}=6 \times 10^{13}$ are not shown because they do not meet our various criteria (Boussinesq, Prandtl window, temperature-drop maximum correction, and minimum temperature difference across the fluid). Nevertheless these extra data for the two experiments keep falling on each other.

In Fig. 4, the smooth surface Nu data are now compensated by $\mathrm{Ra}^{1 / 3}$. To our knowledge, the $1 / 3$ exponent is the highest exponent predicted by the theories of turbulent convection, aside from Kraichnan's regime. Above $\mathrm{Ra}=4$ $\times 10^{11}$, if we fit our data with a local power law, we find an effective exponent of $d \log (\mathrm{Nu}) / d \log (\mathrm{Ra}) \sim 0.4$, significantly larger than the $1 / 3$ prediction. Here again, within the

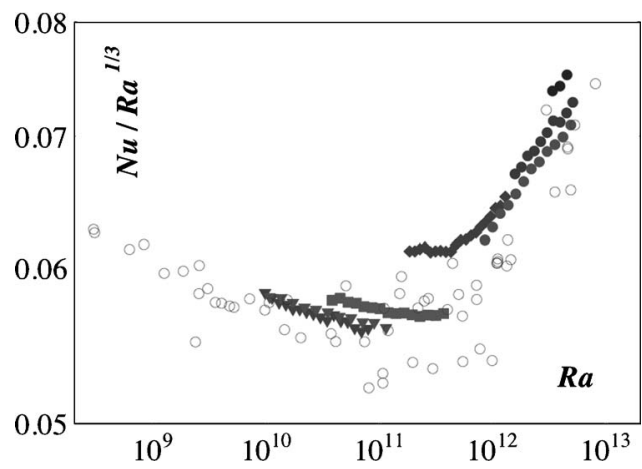

FIG. 4. Compensated Nusselt number $\mathrm{Nu} / \mathrm{Ra}^{1 / 3}$ (on a log scale) vs $\mathrm{Ra}$ for the smooth surface experiments. Open circles: copper bottom plate. Solid symbols: brass bottom plate. experimental uncertainty, the data from both the brass and the all-copper experiments fall on each other.

\section{CONCLUDING REMARKS}

The two experiments presented here are intended to test the Chillà et al. model, ${ }^{10}$ which was able to explain why the ultimate regime is observed in some experiments but not all of them. Within experimental uncertainties, we find that the substitution of a copper plate by its replica made out of brass has no consequence on the global heat transfer, in particular, within the ultimate regime. This result is in clear contradiction with the model.

It seems difficult to adjust the model parameters while preserving its ability to discriminate between the cryogenic experiments. $^{2-5,7}$ This suggests that the critical difference between these experiments has to be searched elsewhere. A candidate is the large scale flow structure (LSFS). Its bimodality, which was evidenced ${ }^{21-23}$ up to $\mathrm{Ra} \sim 10^{12}$, could explain the interesting $\mathrm{Nu}(\mathrm{Ra})$ dependences, near the transition, for both plots of this paper. In 2001, it was conjectured ${ }^{14}$ that this transition to the ultimate regime could only occur for one LSFS, at least below $\mathrm{Ra} \sim 10^{17}$.

\section{ACKNOWLEDGMENTS}

We are indebted to Bernard Castaing and Francesca Chillà for their various inputs in this work and to François Debray and Christophe Trophime for assistance with finite element simulations. This work was supported by the Région Rhône-Alpes, under Contract No. 301491302.

${ }^{1}$ R. Kraichnan, "Turbulent thermal convection at arbitrary Prandtl numbers," Phys. Fluids 5, 1374 (1962).

${ }^{2} \mathrm{X}$. Z. Wu and A. Libchaber, "Scaling relations in thermal turbulence: The aspect-ratio dependence," Phys. Rev. A 45, 842 (1992).

${ }^{3}$ J. J. Niemela, L. Skrbek, K. R. Sreenivasan, and R. J. Donnelly, "Turbulent convection at very high Rayleigh numbers," Nature (London) 404, 837 (2000).

${ }^{4}$ X. Chavanne, F. Chillà, B. Castaing, B. Hébral, B. Chabaud, and J. Chaussy, "Observation of the ultimate regime in Rayleigh-Bénard convection,” Phys. Rev. Lett. 79, 3648 (1997).

${ }^{5}$ J. J. Niemela, L. Skrbek, K. R. Sreenivasan, and R. J. Donnelly, "Confined turbulent convection," J. Fluid Mech. 481, 355 (2003).

${ }^{6} \mathrm{~S}$. Kenjereš and K. Hanjalic, "Numerical insight into flow structure in ultraturbulent thermal convection,” Phys. Rev. E 66, 036307 (2002).

${ }^{7}$ P.-E. Roche, B. Castaing, B. Chabaud, and B. Hébral, "Observation of the $1 / 2$ power law in Rayleigh-Bénard convection," Phys. Rev. E 63, 045303(R) (2001).

${ }^{8}$ L. P. Kadanoff, "Turbulent heat flow: Structures and scaling," Phys. Today 54 (8), 34 (2001).

9J. Sommeria, "The elusive 'ultimate state' of thermal convection," Nature (London) 398, 294 (1999).

${ }^{10}$ F. Chillà, M. Rastello, S. Chaumat, and B. Castaing, "Ultimate regime in Rayleigh-Bénard convection: The role of plates," Phys. Fluids 16, 2452 (2004).

${ }^{11}$ R. Verzicco, "Effects of nonperfect thermal sources in turbulent thermal convection," Phys. Fluids 16, 1965 (2004).

${ }^{12}$ J. C. R. Hunt, A. J. Vrieling, A. J. Nieuwstadt, and H. J. S. Fernando, "Influence of the thermal diffusivity of the lower boundary on eddy motion in convection," J. Fluid Mech. 491, 183 (2003).

${ }^{13}$ X. Chavanne, F. Chillà, B. Chabaud, B. Castaing, and B. Hébral, "Turbulent Rayleigh-Bénard convection in gaseous and liquid He," Phys. Fluids 13, 1300 (2001).

${ }^{14}$ P.-E. Roche, "Convection thermique turbulente en cellule de RayleighBénard cryogénique" Ph.D. thesis, Université Joseph Fourier, Grenoble, 2001. 
${ }^{15}$ R. R. Conte, Eléments de cryogénie (Masson et Cie Editeurs, Paris, 1970). ${ }^{16}$ The distance between grooves previously reported in the copper rough cell was wrong by a factor of 2 . This does not change the interpretation and conclusion of this previous paper, which only relies on the grooves' thickness.

${ }^{17}$ The MEFISTO finite element package has been used. It is available at http://www.ann.jussieu.fr/ perronnet/mefisto.gene.html. Some of the results have been cross-checked with a commercial finite element software (ANSYS).

${ }^{18}$ Model GR-200B-2500, Lake Shore Cryotronics Inc., Westerville, OH.

${ }^{19}$ P.-E. Roche, B. Castaing, B. Chabaud, and B. Hébral, "Heat transfer in turbulent Rayleigh-Bénard convection below the ultimate regime," J. Low Temp. Phys. 134, 1011 (2004).

${ }^{20}$ P.-E. Roche, B. Castaing, B. Chabaud, B. Hébral, and J. Sommeria, "Side wall effects in Rayleigh Bénard experiments," Eur. Phys. J. B 24, 405 (2001). This rough surface cell was built to test a consequence of the Kraichnan theory, stating that in such a cell the logarithmic correction can be "frozen" and the $1 / 2$ power law measured.

${ }^{21}$ P.-E. Roche, B. Castaing, B. Chabaud, and B. Hébral, "Prandtl and Rayleigh numbers dependences in Rayleigh Bénard convection," Europhys. Lett. 58, 693 (2002).

${ }^{22} \mathrm{R}$. Verzicco and R. Camussi, "Numerical experiments on strongly turbulent thermal convection in a slender cylindrical cell," J. Fluid Mech. 477, 19 (2003).

${ }^{23}$ F. Chillà, M. Rastello, S. Chaumat, and B. Castaing, "Long relaxation times and tilt sensitivity in Rayleigh Bénard turbulence," Eur. Phys. J. B 40, 223 (2004). 